\title{
Coupling of deterministically activated quantum emitters in hexagonal boron nitride to plasmonic surface lattice resonances
}

\author{
Nicholas V. Proscia1,2,\#, Robert J. Collison ${ }^{3,4, \#, ~ C a r l o s ~ A . ~ M e r i l e s ~}{ }^{1,2, *}$, \\ Vinod M. Menon ${ }^{1,2, *}$ \\ ${ }^{1}$ Dept. of Physics, CUNY-City College of New York, New York, NY 10031, USA. \\ ${ }^{2}$ Dept. of Physics, CUNY-Graduate Center, New York, NY 10016, USA \\ ${ }^{3}$ Dept. of Chemistry, CUNY-City College of New York, New York, NY 10031, USA
}

${ }^{4}$ Ph. D. Program in Chemistry, The Graduate Center of the City University of New York, New York,

NY, 10016.

\#Equal contribution

*Corresponding authors: cmeriles@ccny.cuny.edu, vmenon@ccny.cuny.edu

\begin{abstract}
Cooperative phenomena stemming from radiation-field-mediated coupling between individual quantum emitters are presently attracting broad interest for on-chip photonic quantum memories and longrange entanglement. Common to these applications is the generation of electro-magnetic modes over macroscopic distances. Much research, however, is still needed before such systems can be deployed in the form of practical devices, starting with the investigation of alternate physical platforms. Quantum emitters in two-dimensional (2D) systems provide an intriguing route because these materials can be adapted to arbitrarily shaped substrates to form hybrid systems where emitters are near-field-coupled to suitable optical modes. Here, we report a scalable coupling method allowing color center ensembles in a van der Waals material - hexagonal boron nitride - to couple to a delocalized high quality plasmonic surface lattice resonance. This type of architecture is promising for photonic applications, especially given the ability of the hexagonal boron nitride emitters to operate as singlephoton sources at room temperature.
\end{abstract}

Keywords: defect, hexagonal boron nitride, surface plasmons, surface lattice resonance, strain, quantum emission, 2D materials, coupling, photoluminescence, delocalization.

\section{Introduction}

Van der Waals materials in the form of thin sheets (1$50 \mathrm{~nm}$ ) or single atomic layers have received a great deal of attention as a light source due to their unique mechanical, electronic and optical properties [1-4].
Particularly, their ability to conform and integrate with other material systems offer unprecedented control over their electronic and optical response [5-8]. Additionally, these material systems exhibit intriguing quantum phenomena both at the macro scale (e.g., room temperature quantum Hall effect [9], condensates [10], and superconductivity [11]) and nanoscale (e.g., single photon emission from color centers [12,13], and excitons [14-17]). These features have given the scientific community new ways to explore quantum phenomena while setting the stage for novel devices.

From among the many van der Waals systems presently attracting attention, here we focus on hexagonal boron nitride ( $\mathrm{hBN}$ ), a semiconductor featuring a wide band gap (of approximately $6 \mathrm{eV}$ [18]) and high thermal conductivity [19]. This material finds a wide range of applications, from catalysis [20] to electronics (e.g., as a component for atomically-thin heterostructures $[21,22])$. Its crystal lattice is isomorphic to graphene, with boron and nitrogen forming the basis elements for a honeycomb structure [23]. hBN was identified recently as the host of room temperature single photon emitters (SPE) associated with point defects in its crystal lattice. These SPEs have properties needed in a single photon source (SPS) which include high excitation and out-coupling efficiency, photon purity, and a low Debye-Waller factor [69]. While the exact nature of the defects is still unclear, the low material cost, room temperature operation, high photon count rates, spectral tunability, and narrow linewidth make these emitters highly attractive for quantum technologies [24-32].

Recent studies have shown that hBN grown via chemical vapor deposition (CVD) can contain a high 
density of point defects that can be "activated" (i.e., transformed into a fluorescent state) either by thermal annealing or substrate-engineering [33,34]. In this latter case, the emitter position (and, to some extent, the areal concentration [35]) can be controlled, hence providing opportunities for integration with photonic structures [36,37].

Processing information with quantum emitters requires scalable methods for deterministically placing these emitters in integrated photonic circuits and subsequently coupling to structures that can direct, amplify, and potentially modulate the emitted photons. Small ensembles of independent emitters coupled to a cavity have recently gained attention with an eye on applications in quantum memory storage and its ability to act as entangled systems $[38,39]$. On the other hand, photonic structures such as plasmonic surface lattice resonances (SLRs) supporting delocalized or propagating modes provide one attractive route to establishing longrange interactions between electronically independent emitters so as to control emission directionality and intensity. Coupling of emitters to this class of photonic systems has attracted recent attention due to its potential as tunable light sources and compact lasing. [4047]

In this report, we focus on engineering the emission properties of room temperature SPSs in hBN by combining deterministic emitter activation and coupling to an optically active substrate. This is achieved by placing 20-nm-thick hBN flakes on silver nano-pillar arrays which support SLRs. Capitalizing on the unique mechanical properties of $2 \mathrm{D}$ materials, we activate the quantum emitters located in a target area by suitably shaping the underlying substrate $[7,8,33]$, and subsequently engineer their emission both directionally and spectrally with the aid of array-supported SLR modes [40]. While the exact mechanism by which the $\mathrm{hBN}$ emitters activated has not been currently isolated, a likely candidate is strain caused by the folding of the $\mathrm{hBN}$ around nanopillars, which in turn would lead the emitter into a bright radiative state $[7,8,33,48]$. The number of emitters can be controlled via the size of the activated area and the intrinsic defect density [34].

\section{Materials and Methods}

\subsection{Sample Preparation fabrication}

In our experiments, we start with the fabrication of arrays of silver nanopillars via e-beam lithography. To this end, a PMMA film (positive resist) was patterned on a glass substrate to produce regular, square arrays of cylindrical holes of variable diameter and separation (pitch). Then, a $100 \mathrm{~nm}$ layer of $\mathrm{Ag}$ was deposited on the patterned PMMA film via electron beam evaporation. Afterwards, a lift-off process was used to remove the Ag-coated PMMA via acetone, leaving behind arrays of silver nanopillars with different diameters and inter-site separations. Subsequently, a $10 \mathrm{~nm}$ conformal coating of alumina was deposited on the sample so as to passivate the $\mathrm{Ag}$ structure as well as to provide the spacing required to prevent silver-induced emitter quenching. To study the interaction between the hBN emission and the plasmonic arrays, we fabricated pillar structures with pitches varying from $220 \mathrm{~nm}$ to 2000 $\mathrm{nm}$ and with pillar diameters from $50 \mathrm{~nm}$ to $400 \mathrm{~nm}$ for each pitch. Generally, pillar arrays with a pillar-to-pillar spacing less than $100 \mathrm{~nm}$ were found to fuse together, hence imposing a practical limit in the type of systems we could probe. Each array was $50 \times 50 \mu \mathrm{m}^{2}$ in size, large enough to allow for a collective SLR mode while still compatible with optical inspection.

The hBN used in the experiment was a CVD-grown 20-nm-thick film commercially available from Graphene Supermarket. The hBN flake had an approximate size of $\sim 5 \times 5 \mathrm{~mm}^{2}$, large enough to cover the entire patterned area with a continuous film. The hBN was deposited on the Ag pillar substrate via a wet transfer protocol described in Ref. [33].

\subsection{Optical characterization}

In order to obtain angle-resolved spectra in Figures $1 \& 4$, the Fourier image of the sample was projected onto the spectrometer slit (Princeton Instruments SpectraPro HR 750). The white light is focused on the array through the excitation objective (Exc. Obj.) and the transmitted light or PL was collected by the collection objective (Coll. Obj.) The collimated transmitted light is then focused by a tube lens (TL) to form a real image plane (IP) that is one focal distance away from the Bertrand lens (BL) where its back focal plane is at the spectrometer slit (Det.) [49]. In this configuration, the spectrometer captures the Fourier image of the $\mathrm{Ag}$ pillar array, where the linear polarizer (Pol.) is used to resolve p- and s-polarizations. For angle-resolved transmission spectra, the sample was illuminated via a tungsten lamp (Olympus) with a 0.6 NA long-working-distance objective (Olympus SLMPLN100x). For the photoluminescence spectra in Fig. 4, a beam size of $\sim 40 \mu \mathrm{m}$ was used to excite the majority of the pillars in a given array.

Photoluminescence (PL) spectra were collected via an infinity-corrected $50 \times, 0.83$ numerical aperture (NA) objective (Olympus). The confocal PL experiments in Fig. 3 were performed with a custom-built confocal microscope. A piezo nano-stage was used to 


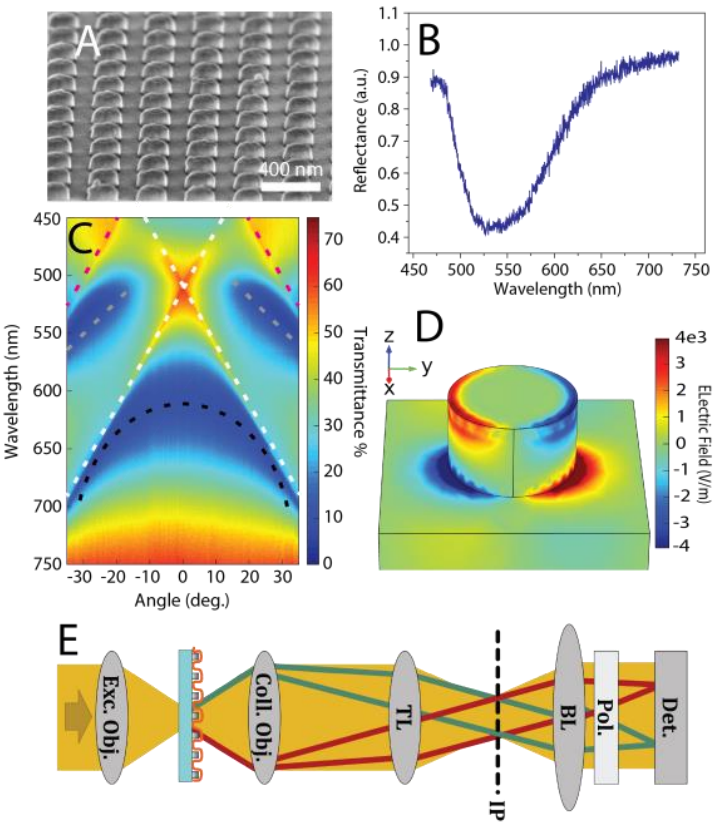

Figure 1: (A) An angled scanning electron microscopy (SEM) image of a bare array of silver nanopillars (345 $\mathrm{nm}$ pitch and $150 \mathrm{~nm}$ pillar diameter). (B) Reflectance spectrum a single pillar from a confocal collection spot. (C) Measured angle-resolved transmission spectrum of the pillar geometry in (A) for $p$-polarized transverse magnetic (TM) incident light. White and red (black and grey) dotted lines correspond to RA (SLR) modes. (D) Simulated $E_{y}$-field mode plot for the array in (A) of the out-ofplane SLR mode at an angle of $25 \mathrm{deg}$. and wavelength of $655 \mathrm{~nm}$. (E) Schematic diagram showing the Fourier microscope used to capture the angle-resolved spectra in (C) and Fig. 4. The Bertrand lens (BL) is placed after tube lens (TL) to form an angle-resolved (signified by the green and red traces) Fourier image at the silt of the detector (spectrometer).

raster scan the sample across a fixed laser and collection path. The focused spot size had a diameter of $\sim 1$ $\mu \mathrm{m}$; the collection spot was $\sim 400 \mathrm{~nm}$. The PL in Fig. 3B was excited via a $510 \mathrm{~nm}, 500 \mathrm{fs}$ pulsed fiber laser with a repetition rate of $80 \mathrm{MHz}$ (Toptica FemtoFiber pro TVIS). The PL for the rest of the experiment was excited via a $460 \mathrm{~nm} \mathrm{CW}$ diode laser.

\section{Results and Discussion}

SLRs are hybrid plasmonic diffractive modes found in one- and two-dimensional periodic arrays of plasmonic nanoparticles. They are formed by the coupling of the localized surface plasmon resonances (LSPRs) of the discrete metal particles to in-plane diffraction orders known as Rayleigh anomalies (RAs) [50]. Fig. 1A shows an SEM image of a typical $\mathrm{Ag}$ nanopillar array recorded at a $70^{\circ}$ tilt angle and a $30^{\circ}$ scan rotation angle. It has a pitch of $345 \mathrm{~nm}$ and a pillar diameter of $150 \mathrm{~nm}$. As seen from the SEM image, the pillars are slightly tapered at the top. This change in diameter can lead to broadening of the LSPR mode as it depends highly on the pillar cross section [40]. Hybridized SLR modes occur at wavelengths slightly red-shifted from the wavelengths of the RAs (the high transmittance lines crossing at $\sim 510 \mathrm{~nm}$ in Fig. 1C). The SLR is seen in the dispersive extinction feature from $575 \mathrm{~nm}$ to $700 \mathrm{~nm}$. The SLR in Fig. 1C probed with a broadband white source has a Fano-resonance

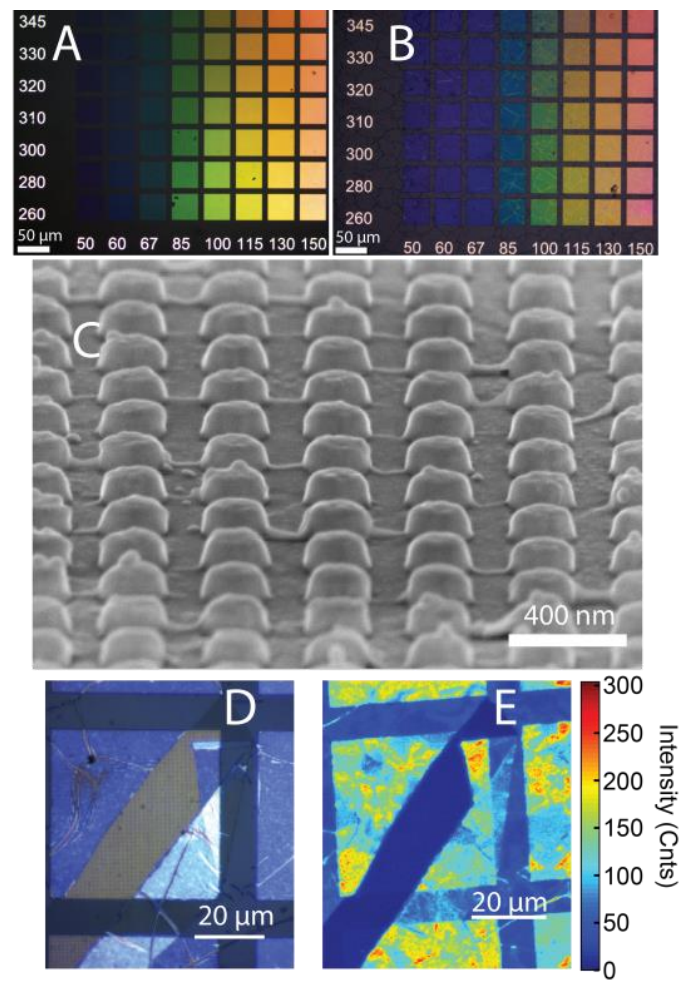

Fig 2. (A) Bright field optical microscopy image of various Ag pillar arrays on a glass substrate. The pitch increases vertically while pillar diameters increase horizontally. The target sizes (in $\mathrm{nm}$ ) are visible along the left and bottom sides of the patterned region. (B) Same as in (A) but after the transfer of a 20-nm-thick sheet of hBN. (C) Angled SEM image of the Ag pillar array covered with $20 \mathrm{~nm}$ hBN film. The hBN film takes the shape of the substrate without tearing. (D) Optical microscope image of partially torn $\mathrm{hBN}$ on the Ag pillar array having $150 \mathrm{~nm}$ diameter pillars and a $470 \mathrm{~nm}$ lattice period. (E) Confocal PL image of the same array as in (D).

line shape due to interference between the scattered light from the SLR mode and the directly transmitted broadband light [51]. The relevant modes here are the so-called 'out-of-plane SLRs', corresponding to LSPRs predominantly localized near the upper and lower ends of the pillars $[52,53]$.

For the geometry in Fig. 1A, the non-dispersive LSPR mode for the $150 \mathrm{~nm}$ pillars, shown in Fig. 1B, splits into two branches. The branch outside the $510 \mathrm{~nm}$ RA has two symmetrical lobes occurring between 500 $\mathrm{nm}$ and $560 \mathrm{~nm}$ centered at the original LSPR mode (indicated by the gray dotted lines in Fig. 1C). These two lobes correspond to the SLR produced by the coupling 

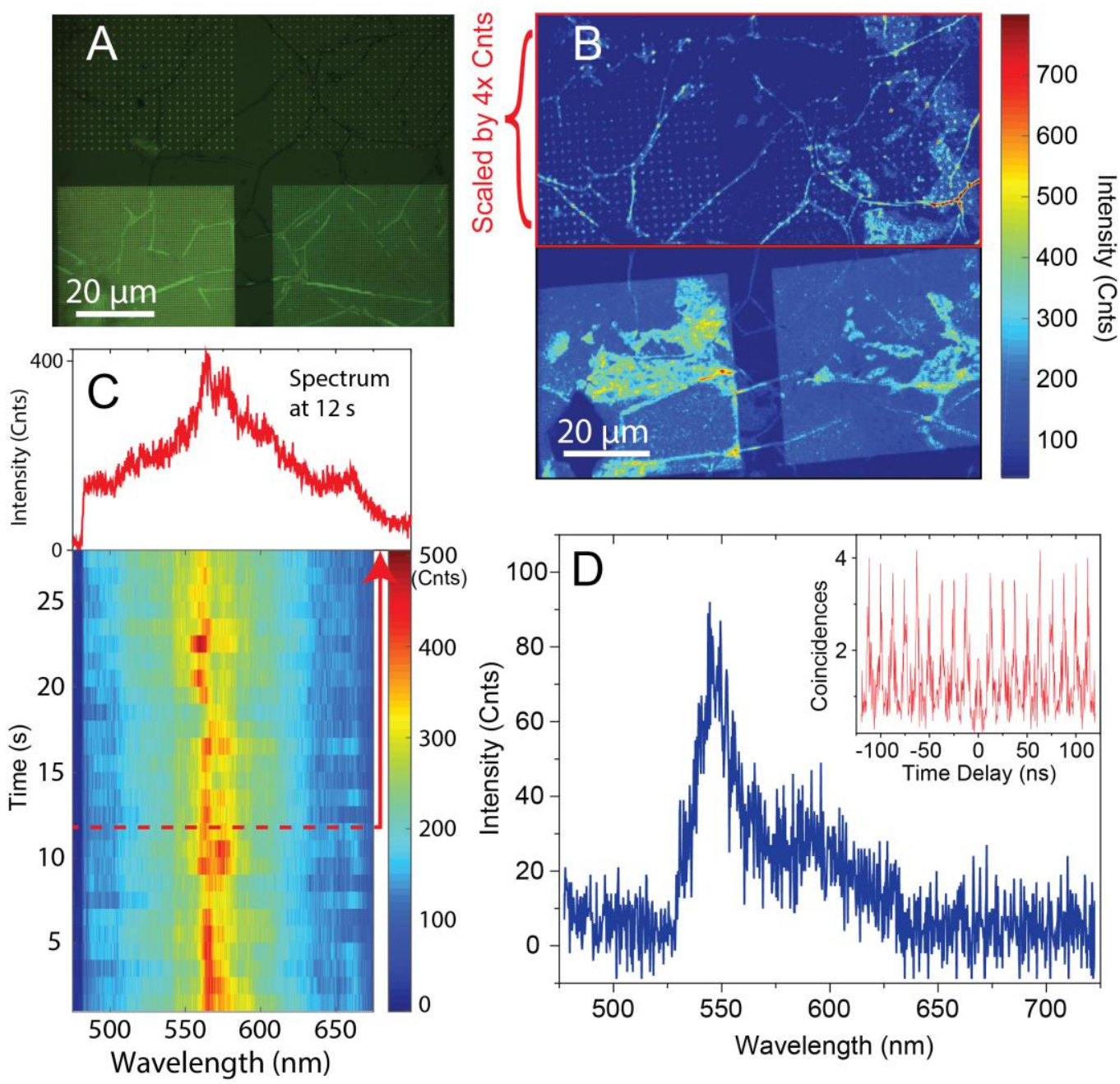

Figure 3. (A) Optical microscope image of hBN-draped Ag pillar arrays. The particle diameters are 400 and $300 \mathrm{~nm}$ for the pair of arrays in the left and the right halves, respectively; the lattice periods are 2 um and $620 \mathrm{~nm}$ for the arrays in the upper and lower halves, respectively. (B) hBN photoluminescence for the same region as in (A). For clarity, we use different color scales for the arrays in the upper and lower halves. (C) Time-resolved emission spectra from a point on the array with $430 \mathrm{~nm}$ pitch and $100 \mathrm{~nm}$ pillar diameter. Several emitters are seen alternating between bright and dark states in the $550-$ $675 \mathrm{~nm}$ spectral band. The upper spectrum (red trace) corresponds to a line cut at $12 \mathrm{~s}$. (D) Spectra from an isolated 100$\mathrm{nm}$-diameter pillar from a $2000-\mathrm{nm}$-pitch array. The phonon sideband is clearly visible $\sim 165 \mathrm{eV}$ from the zero-phonon line at $550 \mathrm{~nm}$ (blue trace). Inset: Photon correlation data via a pulsed Hanbury-Brown-Twiss measurement; an autocorrelation value $g^{(2)}(0)=0.54$ was determined from the curve, implying 2 or less emitters.

of the Ag pillar LPSR to the higher energy $(0, \pm 1)_{\text {air }}$ RAs (red dotted lines). The lower energy $(0, \pm 1)_{\text {glass }} \mathrm{RA}$ modes (white dotted lines) couple to the LSPR such that the resulting SLR (black dotted line) is continuous across normal incidence and follows the dispersion of the RA angles with gradually decreasing linewidths at larger angles. This is the typical behavior of SLRs [52]. The quality of the mode increases at larger angles as these modes are longer lived when they become less localized at the individual Ag pillars

The bright field microscope images of the Ag pillars arrays in Fig. 2 allow us to compare the SLR's optical properties before and after hBN transfer. Beside a $\sim 20 \mathrm{~nm}$ red shift of the modes (expected from the higher index of the hBN film) and a slight decrease in the resonance quality, we find the SLRs were largely unaffected by the hBN film and retained all its core features. We fabricated Ag arrays with variable pitch and pillar diameter as seen in Figs. 2A and 2B. This allows us to study a large variety of SLR resonances as the hBN color centers occur across most of the visible spectrum, centered at $\sim 570 \mathrm{~nm}[33,54]$. The 345-nm-pitch, 150-nm-pillar-diameter array was found to have the highest emission contrast on and off the SLR. Fig. 2E shows a confocal scan of the observed PL. The hBN shows preferential emission when on the pillar array regions, while in the partially torn regions (seen in Fig. 2D) the PL intensity is drastically reduced indicating that the $\mathrm{hBN}$ is the source of the observed PL. 

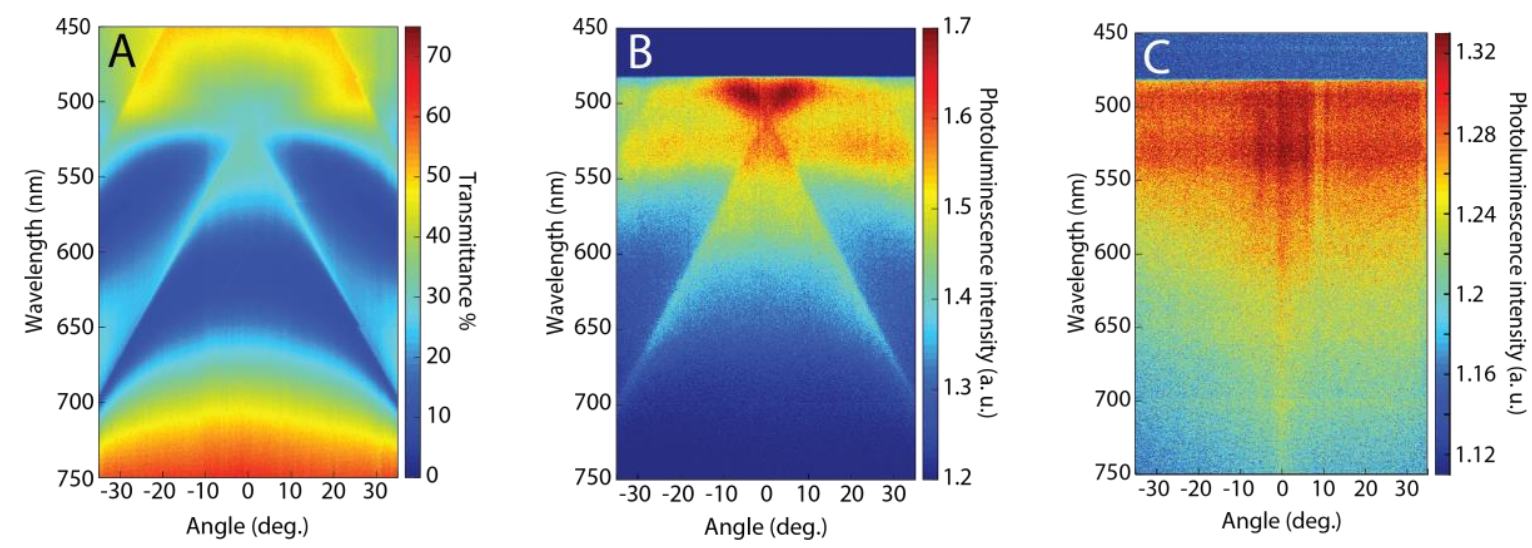

Figure 4: (A) Transverse magnetic (TM) transmission spectrum of 345-nm-period/ 150-nm-pillar-diameter array studied in Fig. 1 but with overlaid hBN. (B) Coupled PL from the hBN film on the same array with illumination from a $460 \mathrm{~nm}$ CW diode laser. (C) Angle-resolved PL spectrum from hBN on the $2000 \mathrm{~nm}$ pitch array with $150 \mathrm{~nm}$ diameter pillars. SLRs in the visible wavelength range are not supported due to the large spacing between the pillars.

Fig. 3 displays the emission characteristics of these hybrid hBN/Ag arrays. The hBN-induced photoluminescence from isolated plasmonic particles is highly localized to individual pillars as seen in the upper quadrants of Figs. 3A and 3B. These arrays have a $2 \mu \mathrm{m}$ spacing and do not support collective SLRs. The pillar locations of all four arrays are clearly identifiable in the bright field image of Fig. 3A. At each pillar site, we can probe individual (or few) emitters as the pillar diameter is made sufficiently small [33]. For illustration purposes, Fig. 3D shows a spectrum for a 100-nm-diameter pillar with 2 or less defects $\left(\mathrm{g}^{(2)}(0)<.54 \pm .04\right)$. The $\mathrm{g}^{(2)}(0)$ was determined from the autocorrelation data in the inset of Fig. 3D by taking the ratio between the area under the coincidence peak at $t=0$ and the average area of $10 t \neq 0$ peaks. The area was determined by fitting each peak with a Lorentzian function [30]. Arrays that support SLRs (lower quadrants in Fig. 3A), on the other hand, become collectively bright as seen in the confocal image of Fig. 3B. As the pitch decreases, emitters get closer to each other and the collected fluorescence contains contributions from several defects. The latter leads to a broad dynamical emission across most of the visible spectrum (line cut in Fig. 3C) with emitters blinking intermittently on a timescale of seconds (contour plot in Fig 3C).

To study the interplay between emitter fluorescence and the SLRs, we look at the optical characteristics of the hBN-covered array already characterized in Fig. 1. Aside from the slight changes pointed out above, the optical transmission spectrum in Fig. 4A shows the modes remain largely unaltered. By contrast, blue laser excitation of the entire array ( $40 \mu \mathrm{m}$ spot size) shows the $\mathrm{hBN}$ emission is drastically modified via coupling into the SLR modes (Fig. 4B). In particular we observe the PL becomes highly directional, largely mirroring the RA-induced SLR dispersion at high angles [40]. Also, the emission spectral band is extended by the SLR mode into the deep red, where the intensity of the defect emission is otherwise an order of magnitude weaker (red spectra of Fig. 3C). All of the above demonstrates good coupling between the defect center emission and the SLR modes. In the absence of an SLR mode (Fig. $4 \mathrm{C})$, the $\mathrm{hBN}$ emission shows no dispersion. This contrast with Fig. $4 \mathrm{~B}$ confirms that the dispersion of the $\mathrm{hBN}$ emission in Fig. 4B is derived from the coupling of the hBN emitters to the SLR mode supported by the $345 \mathrm{~nm}$ pitch Ag nanopillar array.

\section{Conclusions}

In summary, we presented a method for engineering room temperature quantum emission in a scalable platform where the emitters are activated at the array sites via substrate engineering. For suitable array parameters, the defect emission couples to the optically active plasmonic substrate and undergoes a modification in its directionality and spectral intensity. Coupling of emitter systems with photonic structures that support delocalized or propagating modes permits the mutual interaction between these distinct and spatially separated color centers. Such structures could facilitate long range energy transfer between individual emitters. Future work could focus on controlling the number of emitters coupling to the SLR mode via the size of the active region, through the number of intrinsic defects present in the material, or by spectral filtering [34].

Acknowledgements: N.V.P. and V.M.M. acknowledge support from the NSF MRSEC program (DMR-1420634) and the NSF EFRI 2-DARE program (EFMA -1542863). C.A.M acknowledge support from 
the National Science Foundation through grants NSF1619896, NSF-1726573, and from Research Corporation for Science Advancement through a FRED Award. All authors acknowledge support from and access to the infrastructure provided by the NSF CREST IDEALS (NSF grant number HRD-1547830). The fabrication and SEM work was performed at the Advanced Science Research Center NanoFabrication Facility of the Graduate Center at the City University of New York.

\section{References}

1. Y. Liu, N. O. Weiss, X. Duan, H. C. Cheng, Y. Huang, and X. Duan, "Van der Waals heterostructures and devices," Nat. Rev. Mater. 1, (2016).

2. M. Koperski, M. R. Molas, A. Arora, K. Nogajewski, A. O. Slobodeniuk, C. Faugeras, and M. Potemski, "Optical properties of atomically thin transition metal dichalcogenides: observations and puzzles," Nanophotonics 6, 1289-1308 (2017).

3. M. F. El-Kady, Y. Shao, and R. B. Kaner, "Graphene for batteries, supercapacitors and beyond," Nat. Rev. Mater. 1, (2016).

4. D. S. Schulman and S. Das, "The Hope of Two-Dimensional Heterostructures: A review of recent breakthroughs," IEEE Nanotechnol. Mag. (2017).

5. X. Liu, T. Galfsky, Z. Sun, F. Xia, E.-C. Lin, Y.-H. Lee, S. Kéna-Cohen, and V. M. Menon, "Strong light-matter coupling in two-dimensional atomic crystals," Nat. Photonics 9, (2015).

6. G. D. Shepard, O. A. Ajayi, X. Li, X. Y.Zhu, J. Hone, and S. Strauf, "Nanobubble induced formation of quantum emitters in monolayer semiconductors," 2D Mater. 4, (2017).

7. C. Palacios-Berraquero, D. M. Kara, A. R. P. Montblanch, M. Barbone, P. Latawiec, D. Yoon, A. K. Ott, M. Loncar, A. C. Ferrari, and M. Atatüre, "Large-scale quantumemitter arrays in atomically thin semiconductors," Nat. Commun. 8, 1-6 (2017).

8. A. Branny, S. Kumar, R. Proux, and B. D. Gerardot, "Deterministic strain-induced arrays of quantum emitters in a twodimensional semiconductor," Nat. Commun. 8, 1610.01406v1 (2016).

9. K. S. Novoselov, Z. Jiang, Y. Zhang, S. V Morozov, H. L. Stormer, U. Zeitler, J. C. Maan, G. S. Boebinger, P. Kim, and A. K. Geim, "Room-temperature quantum Hall effect in graphene.," Science 315, 1379 (2007).
10. B. Debnath, Y. Barlas, D. Wickramaratne, M. R. Neupane, and R. K. Lake, "Exciton condensate in bilayer transition metal dichalcogenides: Strong coupling regime," Phys. Rev. B 96, 174504 (2017).

11. Y. Cao, V. Fatemi, S. Fang, K. Watanabe, T. Taniguchi, E. Kaxiras, and P. JarilloHerrero, "Unconventional superconductivity in magic-angle graphene superlattices," Nat. Publ. Gr. 556, (2018).

12. T. T. Tran, K. Bray, M. J. Ford, M. Toth, and I. Aharonovich, "Quantum Emission From Hexagonal Boron Nitride Monolayers," Nat. Nanotechnol. 11, 1-12 (2015).

13. L. Museur, E. Feldbach, and A. Kanaev, "Defect-related photoluminescence of hexagonal boron nitride," Phys. Rev. B Condens. Matter Mater. Phys. 78, 1-8 (2008).

14. P. Tonndorf, R. Schmidt, R. Schneider, J. Kern, M. Buscema, G. a. Steele, A. Castellanos-Gomez, H. S. J. van der Zant, S. Michaelis de Vasconcellos, and R. Bratschitsch, "Single-photon emission from localized excitons in an atomically thin semiconductor," Optica 2, 347 (2015).

15. Y. He, G. Clark, J. R. Schaibley, Y. He, M. Chen, Y. Wei, X. Ding, Q. Zhang, W. Yao, X. Xu, C. Lu, and J. Pan, "Single quantum emitters in monolayer semiconductors," Nat. Nanotechnol. 10, 497-502 (2015).

16. A. Srivastava, M. Sidler, A. V Allain, D. S. Lembke, A. Kis, and A. Imamo, "Optically active quantum dots in monolayer WSe 2," Nat. Nanotechnol. 10, 491-496 (2015).

17. M. Koperski, K. Nogajewski, A. Arora, V. Cherkez, P. Mallet, J. Veuillen, J. Marcus, P. Kossacki, and M. Potemski, "Single photon emitters in exfoliated WSe 2 structures," Nat. Nanotechnol. 10, 503-506 (2015).

18. G. Cassabois, P. Valvin, and B. Gil, "Hexagonal boron nitride is an indirect bandgap semiconductor," Nat. Photonics 10, 262-267 (2016).

19. D. Golberg, Y. Bando, Y. Huang, T. Terao, M. Mitome, C. Tang, and C. Zhi, "Boron nitride nanotubes and nanosheets," ACS Nano 4, 2979-2993 (2010).

20. J. T. Grant, C. A. Carrero, F. Goeltl, J. Venegas, P. Mueller, S. P. Burt, S. E. Specht, W. P. Mcdermott, A. Chieregato, and I. Hermans, "Selective oxidative dehydrogenation of propane to propene using boron nitride catalysts," Science 354, 1570-1573 (2016).

21. W. Pan, J. Xiao, J. Zhu, C. Yu, G. Zhang, Z. Ni, K. Watanabe, T. Taniguchi, Y. Shi, and $\mathrm{X}$. Wang, "Biaxial compressive strain engineering in graphene/boron nitride 
heterostructures.," Sci. Rep. 2, 893 (2012).

22. A. K. Geim and I. V. Grigorieva, "Van der Waals heterostructures.," Nature 499, 419425 (2013).

23. H. Fang, S. L. Bai, and C. P. Wong, "“White graphene" - hexagonal boron nitride based polymeric composites and their application in thermal management," Compos. Commun. 2, 19-24 (2016).

24. T. T. Tran, C. Zachreson, A. M. Berhane, K. Bray, R. G. Sandstrom, L. H. Li, T. Taniguchi, K. Watanabe, I. Aharonovich, and M. Toth, "Quantum Emission from Defects in Single-Crystalline Hexagonal Boron Nitride," Phys. Rev. Appl. 5, 2-6 (2016).

25. N. R. Jungwirth, B. Calderon, Y. Ji, M. G. Spencer, M. E. Flatt, and G. D. Fuchs, "Temperature Dependence of Wavelength Selectable Zero-Phonon Emission from Single Defects in Hexagonal Boron Nitride," Nano Lett. 16, 6052-6057 (2016).

26. X. Li, G. D. Shepard, A. Cupo, N. Camporeale, K. Shayan, Y. Luo, V. Meunier, and S. Strauf, "Nonmagnetic Quantum Emitters in Boron Nitride with Ultranarrow and Sideband-Free Emission Spectra," ACS Nano 11, (2017).

27. S. Choi, T. T. Tran, C. Elbadawi, C. Lobo, X. Wang, S. Juodkazis, G. Seniutinas, M. Toth, and I. Aharonovich, "Engineering and Localization of Quantum Emitters in Large Hexagonal Boron Nitride Layers," ACS Appl. Mater. Interfaces 8, 29642-29648 (2016).

28. L. J. Martinez, T. Pelini, V. Waselowski, J. R. Maze, B. Gil, G. Cassabois, and V. Jacques, "Efficient single photon emission from a high-purity hexagonal boron nitride crystal," Phys. Rev. B - Condens. Matter Mater. Phys. 94, 1-5 (2016).

29. T. T. Tran, D. Wang, Z. Q. Xu, A. Yang, M. Toth, T. W. Odom, and I. Aharonovich, "Deterministic Coupling of Quantum Emitters in 2D Materials to Plasmonic Nanocavity Arrays," Nano Lett. 17, 26342639 (2017).

30. G. Grosso, H. Moon, B. Lienhard, S. Ali, D. K. Efetov, M. M. Furchi, P. Jarillo-Herrero, M. J. Ford, I. Aharonovich, and D. Englund, "Tunable and high-purity room temperature single-photon emission from atomic defects in hexagonal boron nitride," Nat. Commun. 8, 1-8 (2017).

31. M. Nguyen, S. Kim, T. T. Tran, Z. Q. Xu, M. Kianinia, M. Toth, and I. Aharonovich, "Nanoassembly of quantum emitters in hexagonal boron nitride and gold nanospheres," Nanoscale 10, 2267-2274
(2018).

32. T. Vogl, R. Lecamwasam, B. C. Buchler, Y. Lu, and P. K. Lam, "Compact CavityEnhanced Single-Photon Generation with Hexagonal Boron Nitride," ACS Photonics 6, 51 (2019).

33. N. V Proscia, Z. Shotan, H. Jayakumar, P. Reddy, C. Cohen, M. Dollar, A. Alkauskas, M. Doherty, C. A. Meriles, and V. M. Menon, "Near-deterministic activation of room-temperature quantum emitters in hexagonal boron nitride," Optica 5, 1128 (2018).

34. N. Mendelson, Z. Q. Xu, T. T. Tran, M. Kianinia, J. Scott, C. Bradac, I. Aharonovich, and M. Toth, "Engineering and Tuning of Quantum Emitters in FewLayer Hexagonal Boron Nitride," ACS Nano 13, 15 (2019).

35. A. Javey, J. S. Kang, S. B. Desai, J. Guo, G. Seol, R. Kapadia, J. W. Ager, C. Battaglia, and H. Fang, "Strain-Induced Indirect to Direct Bandgap Transition in Multilayer WSe 2," Nano Lett. 14, 4592-4597 (2014).

36. T. Cai, J. H. Kim, Z. Yang, S. Dutta, S. Aghaeimeibodi, and E. Waks, "Radiative Enhancement of Single Quantum Emitters in WSe2 Monolayers Using Site-Controlled Metallic Nanopillars," ACS Photonics 5, 3466-3471 (2018).

37. Y. Luo, G. D. Shepard, J. V. Ardelean, D. A. Rhodes, B. Kim, K. Barmak, J. C. Hone, and S. Strauf, "Deterministic coupling of sitecontrolled quantum emitters in monolayer WSe 2 to plasmonic nanocavities," Nat. Nanotechnol. 13, (2018).

38. T. Zhong, J. M. Kindem, J. Rochman, and A. Faraon, "Interfacing broadband photonic qubits to on-chip cavity-protected rare-earth ensembles," Nat. Commun. 8, 1-7 (2017).

39. R. E. Evans, M. K. Bhaskar, D. D. Sukachev, C. T. Nguyen, A. Sipahigil, M. J. Burek, B. Machielse, G. H. Zhang, A. S. Zibrov, E. Bielejec, H. Park, M. Lončar, and M. D. Lukin, "Photon-mediated interactions between quantum emitters in a diamond nanocavity," Science 665, 662-665 (2018).

40. G. Vecchi, V. Giannini, and J. Gómez Rivas, "Shaping the fluorescent emission by lattice resonances in plasmonic crystals of nanoantennas," Phys. Rev. Lett. 102, (2009).

41. R. Guo, S. Derom, A. I. Väkeväinen, R. J. A. Van Dijk-Moes, P. Liljeroth, D. Vanmaekelbergh, and P. Törmä, "Controlling quantum dot emission by plasmonic nanoarrays," Opt. Express 23, 28206 (2015).

42. W. Zhou, M. Dridi, J. Y. Suh, C. H. Kim, D. T. Co, M. R. Wasielewski, G. C. Schatz, and 
T. W. Odom, "Lasing action in strongly coupled plasmonic nanocavity arrays," Nat. Nanotechnol. 8, 506-511 (2013).

43. A. Yang, T. B. Hoang, M. Dridi, C. Deeb, M. H. Mikkelsen, G. C. Schatz, and T. W. Odom, "Real-time tunable lasing from plasmonic nanocavity arrays," Nat. Commun. 6, (2015).

44. D. Wang, A. Yang, W. Wang, Y. Hua, R. D. Schaller, G. C. Schatz, and T. W. Odom, "Band-edge engineering for controlled multi-modal nanolasing in plasmonic superlattices," Nat. Nanotechnol. 12, 889894 (2017).

45. T. K. Hakala, H. T. Rekola, A. I. Väkeväinen, J.-P. Martikainen, M. Nečada, A. J. Moilanen, and \& P. Törmä, "Lasing in dark and bright modes of a finite-sized plasmonic lattice," Nat. Commun. 8, (2017).

46. S. Schwarz, A. Kozikov, F. Withers, J. K. Maguire, A. P. Foster, S. Dufferwiel, L. Hague, M. N. Makhonin, L. R. Wilson, A. K. Geim, K. S. Novoselov, and A. I. Tartakovskii, "Electrically pumped singledefect light emitters in WSe 2," 2D Mater. 3, 025038 (2016).

47. T. Vogl, R. Lecamwasam, B. C. Buchler, Y. $\mathrm{Lu}$, and P. K. Lam, Space-Compatible Cavity-Enhanced Single-Photon Generation with Hexagonal Boron Nitride (n.d.).

48. Y.-T. Wang, W. Liu, Z.-P. Li, S. Yu, Z.-J. Ke, Y. Meng, J.-S. Tang, C.-F. Li, and G.-C. Guo, "A bubble-induced ultrastable and robust single-photon emitter in hexagonal boron nitride," Arxiv Prepr. 1906.00493, (2019).

49. J. A. Kurvits, M. Jiang, and R. Zia, "Comparative analysis of imaging configurations and objectives for Fourier microscopy," J. Opt. Soc. Am. A 32, 2082 (2015).

50. V. G. Kravets, A. V Kabashin, W. L. Barnes, and A. N. Grigorenko, "Plasmonic Surface Lattice Resonances: A Review of Properties and Applications," Chem. Rev. 118, 59125951 (2018).

51. B. Auguié and W. L. Barnes, "Collective resonances in gold nanoparticle arrays," Phys. Rev. Lett. 101, (2008).

52. V. G. Kravets, F. Schedin, and A. N. Grigorenko, "Extremely narrow plasmon resonances based on diffraction coupling of localized plasmons in arrays of metallic nanoparticles," Phys. Rev. Lett. 101, (2008).

53. W. Zhou, Y. Hua, M. D. Huntington, and T. W. Odom, "Delocalized lattice plasmon resonances show dispersive quality factors," J. Phys. Chem. Lett. 3, 1381-1385 (2012).

54. M. Koperski, K. Nogajewski, and M.
Potemski, "Single photon emitters in boron nitride: More than a supplementary material," Opt. Commun. 411, 158-165 (2018). 